University of Wollongong

Research Online

Faculty of Engineering and Information

Faculty of Engineering and Information

Sciences - Papers: Part A

Sciences

$1-1-2013$

\title{
Energy-loss rate of a fast particle in two-dimensional semiconductors with Rashba spin-orbit coupling
}

Wei Feng

Jiangsu University, wfeng@uow.edu.au

Asya Tawfiq

University of Wollongong, aat495@uowmail.edu.au

J.C Cao

Shanghai Institute

Chao Zhang

University of Wollongong, czhang@uow.edu.au

Follow this and additional works at: https://ro.uow.edu.au/eispapers

Part of the Engineering Commons, and the Science and Technology Studies Commons

Research Online is the open access institutional repository for the University of Wollongong. For further information contact the UOW Library: research-pubs@uow.edu.au 


\title{
Energy-loss rate of a fast particle in two-dimensional semiconductors with Rashba spin-orbit coupling
}

\author{
Abstract \\ The energy-loss rate (ELR) of a charged particle in a two-dimensional semiconductor with Rashba spin- \\ orbit coupling is studied. Our model takes into account of the temperature and density dependence of the \\ electronic properties of the Rashba system. The energy and temperature dependence of the ELR are \\ presented. It is found that a finite Rashba spin-orbit coupling offers a mechanism of tuning the mean \\ scattering time in narrow-gap semiconductors. With a change of Rashba parameter of around 3 times, the \\ mean scattering time can change by one to two orders of magnitude. (C) 2013 American Institute of \\ Physics.
}

\section{Keywords}

dimensional, two, particle, fast, rate, loss, rashba, energy, semiconductors, spin, orbit, coupling

\section{Disciplines}

Engineering | Science and Technology Studies

\section{Publication Details}

Feng, W., Tawfiq, A., Cao, J. \& Zhang, C. (2013). Energy-loss rate of a fast particle in two-dimensional semiconductors with Rashba spin-orbit coupling. Applied Physics Letters, 102 (5), 052113-1-052113-3. 


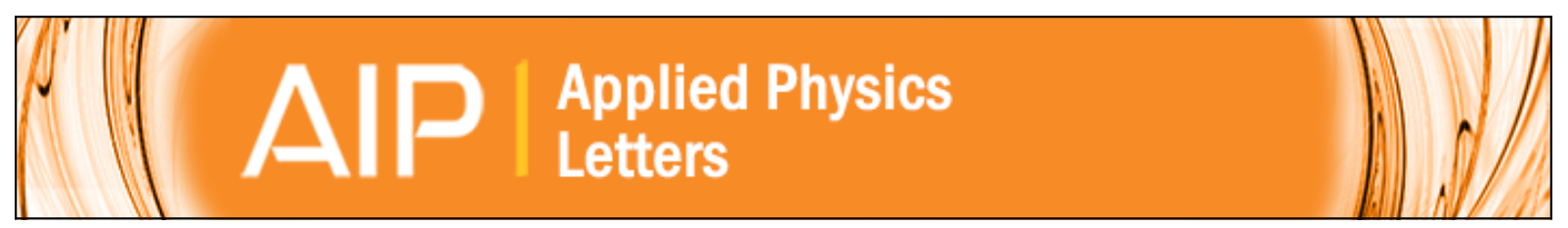

Energy-loss rate of a fast particle in two-dimensional semiconductors with Rashba spin-orbit coupling

W. Feng, Asya Tawfiq, J. C. Cao, and C. Zhang

Citation: Applied Physics Letters 102, 052113 (2013); doi: 10.1063/1.4790847

View online: http://dx.doi.org/10.1063/1.4790847

View Table of Contents: http://scitation.aip.org/content/aip/journal/apl/102/5?ver=pdfcov

Published by the AIP Publishing

\section{Advertisement:}
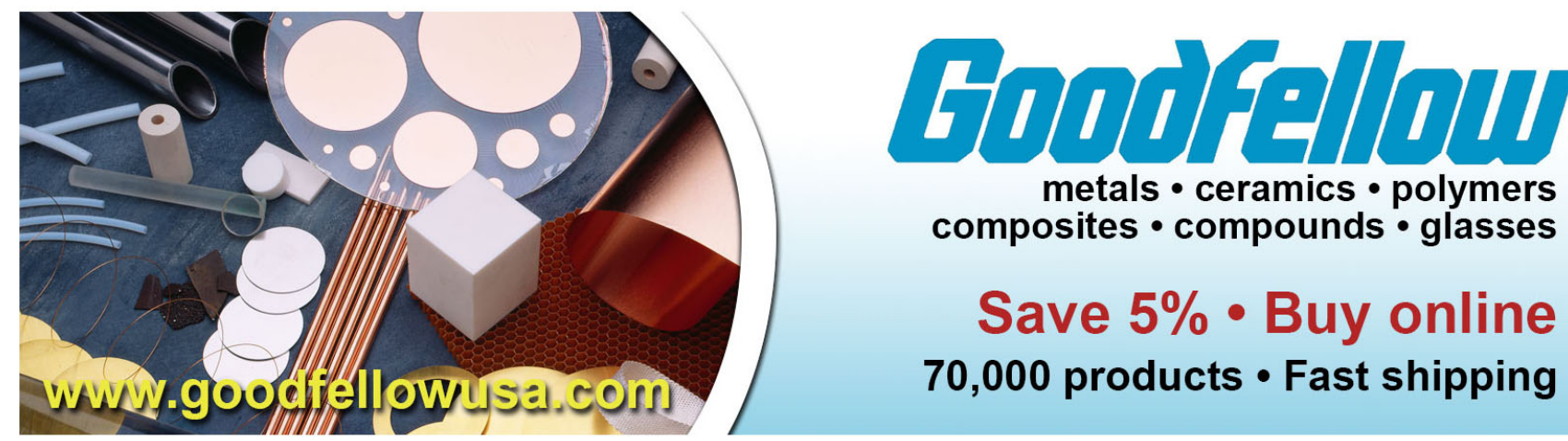

metals • ceramics $\cdot$ polymers composites • compounds • glasses

Save $5 \% \cdot$ Buy online 70,000 products $\cdot$ Fast shipping 


\title{
Energy-loss rate of a fast particle in two-dimensional semiconductors with Rashba spin-orbit coupling
}

\author{
W. Feng, ${ }^{1}$ Asya Tawfiq, ${ }^{2}$ J. C. Cao, ${ }^{3}$ and C. Zhang ${ }^{4, a)}$ \\ ${ }^{1}$ Department of Physics, Jiangsu University, Zhenjiang, Jiangsu, China \\ ${ }^{2}$ School of Physics, University of Wollongong, New South Wales 2522, Australia \\ ${ }^{3}$ Key Laboratory of Terahertz Solid State Technology, Shanghai Institute of Microsystem and \\ Information Technology, Chinese Academy of Sciences, Shanghai, China \\ ${ }^{4}$ School of Physics and Institute for Superconducting and Electronic Materials, University of Wollongong, \\ New South Wales 2522, Australia
}

(Received 5 January 2013; accepted 25 January 2013; published online 6 February 2013)

\begin{abstract}
The energy-loss rate (ELR) of a charged particle in a two-dimensional semiconductor with Rashba spin-orbit coupling is studied. Our model takes into account of the temperature and density dependence of the electronic properties of the Rashba system. The energy and temperature dependence of the ELR are presented. It is found that a finite Rashba spin-orbit coupling offers a mechanism of tuning the mean scattering time in narrow-gap semiconductors. With a change of Rashba parameter of around 3 times, the mean scattering time can change by one to two orders of magnitude. (C) 2013 American Institute of Physics. [http://dx.doi.org/10.1063/1.4790847]
\end{abstract}

The recent advancement in spin-electronics (spintronics) and spintronic materials ${ }^{1}$ has provided opportunities in developing unique optoelectronic devices whose optical and transport properties can be tuned by the intrinsic spin-orbit interaction. Practical spintronic material systems have been realized, and advanced electronic devices have been proposed, such as spin transistors, ${ }^{2}$ spin waveguides, ${ }^{3}$ and spin filters. ${ }^{4}$ In narrow-gap semiconductor nanostructures such as InAs- and $\operatorname{~In~}_{1-x} \mathrm{Ga}_{x}$ As quantum wells, the inversion asymmetry of the confining potential due to the presence of the heterojuction $^{5}$ resulted in the spin splitting (or spontaneous spin splitting) of the carriers in the absence of any applied magnetic field. This effect is similar to that under an inhomogeneous surface electric field. Therefore the inhomogeneous heterostructure confining potential is an electrical equivalence to the Rashba spin splitting or Rashba effect. ${ }^{6}$ This confining potential can be further tuned with an applied gate voltage. Phenomena related to Rashba effects in, e.g., InAs- and $\mathrm{In}_{1-x} \mathrm{Ga}_{x} \mathrm{As}$-based two-dimensional electron gas (2DEG) systems, have been observed. ${ }^{7,8}$ The value of the Rashba parameter in these semiconductor systems can be as high as $4 \times 10^{-11} \mathrm{eV} \mathrm{m}$. The experimental results ${ }^{8}$ have shown that in InAs-and $\mathrm{In}_{1-x} \mathrm{Ga}_{x} \mathrm{As}$-based 2DEG systems, the Rashba effect [with SU(2) symmetry] is responsible for the spontaneous spin splitting. Other contributions such as the Dresselhaus term can also be significant in systems with wide gaps, because it comes mainly from the bulk-inversion asymmetry of the material. ${ }^{9}$ The Rashba effect in a 2 DEG has been investigated by using charge and spin transport. ${ }^{7,8,10}$ It has been shown recently that a random Rashba field can result in nonlinear anomalous Hall effect and negative magnetoresistance. ${ }^{11}$ A strong Rashba coupling gives rise to the intrinsic instability of electronic interface. ${ }^{12}$ A quantum pump based on the Rashba coupling in graphene has been proposed. ${ }^{13}$ Spin Hall effect in a kagome lattice driven by Rashba spinorbit interaction has also been demonstrated theoretically. ${ }^{14,15}$

${ }^{\text {a)} E l e c t r o n i c ~ m a i l: ~ c z h a n g @ u o w . e d u . a u . ~}$
Electron energy loss spectroscopy (EELS) is a powerful tool which provides physical insights on the electronic band structure, phonon excitation, plasmon excitation, and surface properties of a material. It has recently been utilized in the experimental study of the graphene plasmon properties. ${ }^{16,17}$ In order to fully exploit the results of EELS, it is crucial to understand the energy loss rate (ELR) of an external particle in a two-dimensional semiconductor with Rashba spin-orbit coupling (2DS/RSO). The ELR of a particle in marginal Fermi liquid $^{18}$ and ELR of positron in metal ${ }^{19}$ has been theoretically studied. In this work, we investigate ELR of a charged particle in 2DS/RSO. In particular we shall show that the Rashba spin-orbit couple (SOC) offers an additional mechanism for tuning the transport and scattering of a charged particle in semiconductors. The ELR of a charged particle in 2DS/RSO is calculated under the framework of self-consistent field approximation.

The Hamiltonian of a two-dimensional electronic system in the $\mathrm{x}-\mathrm{y}$ plane in narrow-gap semiconductor nanostructures (e.g., InGaAs/InAlAs quantum wells) is given as

$$
H_{0}=\frac{1}{2 m^{*}}\left(p_{x}^{2}+p_{y}^{2}\right)+\frac{\lambda}{\hbar}\left(\sigma_{y} p_{x}-\sigma_{x} p_{y}\right),
$$

where $m^{*}$ is the electron effective mass in the absence of electron-phonon interaction and SOI, $\lambda$ is the Rashba SOI parameter, $\sigma_{x}$ and $\sigma_{y}$ are Pauli spin matrices, and $p_{x}$ and $p_{y}$ are the electron momentum operators. The wavefunction can be written in the form of $\psi(x, y)=u_{\mathbf{k}}(x, y) \xi_{k, \lambda}$, where $u_{\mathbf{k}}(x, y)$ $=\exp \left(i k_{x} x+i k_{y} y\right)$. The eigenvalue is given as

$$
\epsilon_{s, k}=\frac{\hbar^{2} k^{2}}{2 m^{*}}+s \lambda k
$$

where $k=\sqrt{k_{x}^{2}+k_{y}^{2}}$ and $s= \pm 1$, and eigenfunctions $\xi_{k, s}$ are

$$
\xi_{k, s}=\frac{1}{\sqrt{2}}\left(\begin{array}{c}
1 \\
-s\left(k_{y}-i k_{x}\right) / k
\end{array}\right) .
$$


The polarizability function can be evaluated from the bare bubble diagrams and is given by

$$
\Pi(q, \omega)=\sum_{\boldsymbol{k}, s, s^{\prime}} F_{s, s^{\prime}}\left(\theta_{\boldsymbol{k}, \boldsymbol{k}+\boldsymbol{q}}\right) \frac{f_{\boldsymbol{k}+\boldsymbol{q}, s^{\prime}}-f_{\boldsymbol{k}, s}}{\epsilon_{s, \mathbf{k}+\mathbf{q}}-\epsilon_{s, \mathbf{k}}-\omega^{\prime}},
$$

where $\omega^{\prime}=\omega+i \delta$ with $\delta \rightarrow 0$. The wavefunction overlap is $F_{s, s^{\prime}}\left(\theta_{\boldsymbol{k}, \boldsymbol{k}+\boldsymbol{q}}\right)=\left(1+s s^{\prime} \cos \theta_{\boldsymbol{k}, \boldsymbol{k}+\boldsymbol{q}}\right) / 2$, where $\theta_{\boldsymbol{k}, \boldsymbol{k}+\boldsymbol{q}}$ is the angle between $\boldsymbol{k}$ and $\boldsymbol{k}+\boldsymbol{q}$ and $f_{\boldsymbol{k}, s}$ is the Fermi-Dirac distribution function.

We consider a particle with initial momentum $\boldsymbol{p}$ and energy $\varepsilon_{p}$ which is fired onto a $2 \mathrm{DS} / \mathrm{RSO}$. The particle transfers a momentum of $\Delta \mathbf{p}=\boldsymbol{q}$ and energy $\Delta \varepsilon=\omega=\varepsilon_{p}-\varepsilon_{p-q}$ to the RSO/2DS and emerges with a final momentum of $\boldsymbol{p}-\boldsymbol{q}$ and energy $\varepsilon_{p-q}(\hbar \triangleq 1)$. For a particle interacting with the 2DS/ $\mathrm{RSO}$, the energy exchange is determined by $\omega=p q \cos \phi / m$ $-q^{2} / 2 m$, where $\phi$ is the angle between $\boldsymbol{p}$ and $\boldsymbol{q}$ and $m$ is the particle mass. The energy loss rate can be written as

$$
\frac{d \varepsilon_{\mathbf{p}}}{d t}=\int_{0}^{\Lambda} \frac{d^{2} q}{(2 \pi)^{2}} W_{q}(\omega)\left(\varepsilon_{\mathbf{p}+\mathbf{q}}-\varepsilon_{\mathbf{p}}\right)
$$

where $W_{q}(\omega)$ is the transition probability and is given by

$$
W_{q}(\omega)=\frac{2 g_{q}}{1-e^{-\beta \omega}} \operatorname{Im}\left[\frac{1}{D_{q}(\omega)}\right],
$$

where $g_{q}$ is the coupling constant representing the interaction between the incident particle and 2DS/RSO. It is taken to be the screened Coulomb interaction

$$
g_{q}=\frac{2 \pi e^{2}}{\epsilon_{s} \sqrt{q^{2}+q_{s}^{2}}} .
$$

Here $q_{s}^{2}=0.66 r_{s} k_{F}^{2}$ is the screen wave number and $r_{s}=m^{*} e^{2} / \epsilon_{s} k_{F}$ is the plasma parameter, $\epsilon_{s}$ is the static dielectric constant of the host semiconductor, $\beta$ is the inverse temperature $\left(k_{B} T\right)^{-1}$, and $D_{q}(\omega)$ is the dielectric function. The dielectric function under random-phase-approximation is given as

$$
D_{q}(\omega)=1-\nu_{q} \Pi(q, \omega),
$$

where $\nu_{q}$ is the strength of electron-electron interaction of 2DS/RSO. The imaginary part of the inverse dielectric function in Eq. (6) signifies the incident particle energy-loss in 2DS/RSO. It is straight forward to see that

$$
\operatorname{Im}\left[\frac{1}{D_{q}(\omega)}\right]=\frac{\nu_{q}}{\left|D_{q}(\omega)\right|^{2}} \operatorname{Im}[\Pi(q, \omega)] .
$$

Here the plasmon oscillation has been ignored since it is heavily damped in 2DS/RSO for large momentum scattering. The integration upper limit $\Lambda$ is determined by the maximum momentum change of the fast particle in an elastic back scattering, which is $2 p$. The chemical potential of the $2 \mathrm{DS} / \mathrm{RSO}$ is determined self-consistently from the charge density at arbitrary temperatures.

In Fig. 1, we show the dependence of ELR on the momentum of the incoming particle for various Rashba SOI

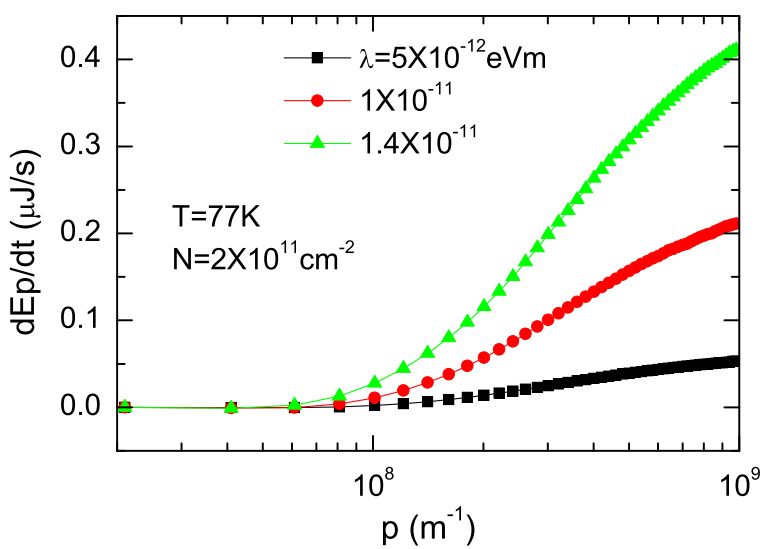

FIG. 1. ELR as a function of momentum of the incoming electron at $\mathrm{T}=77 \mathrm{~K}$ under several different Rashba spin-orbit coupling parameters.

strengths. At fixed $\lambda$, ELR increases with $p$, a property directly related to the increased phase space when $p$ increases. However, if $p$ is sufficiently large, the ELR is limited by the single particle excitation of 2DS/RSO. Therefore ELR saturates at large $p$. The most interesting feature we observe here is the dependence of ELR on the SOI parameter $\lambda$. As $\lambda$ increases, ELR increases remarkably. This behavior can be understood as follows. In the absence of the spin splitting, there is a single value of momentum transfer corresponding to the transition energy for a photon absorption. Due to Rashba splitting, there are four different momentum transfers for a given frequency, two intra- and two inter-level transitions. This leads to the fine structures in both the real and imaginary part of the dielectric function. These fine structures are only resolved if $\lambda$ is sufficiently large. Under finite $\lambda$, there are two energy branches $(s= \pm 1)$. The electron concentration in the $+(-)$ branch increases (decreases) with $\lambda$. The total excitation spectrum ${ }^{10} \operatorname{Im}\left[\frac{1}{D_{q}(\omega)}\right]$ increases for $q<k_{F}$ and decreases for $q>k_{F}$. The ELR is controlled by $g_{q}$ which decreases rapidly with $q$. As a result, ELR increases with $\lambda$.

The temperature dependent ELR is shown in Fig. 2 for a Rashba parameter of $10^{-11} \mathrm{eVm}$ under different charge concentrations. The temperature dependence is mainly determined by two factors (i) the chemical potential decrease with the temperature and (ii) the thermal phase factor related to the space of electronic transition $(1-\exp (-\beta \omega))^{-1}$. The first effect of weak as there is only a small correction to the

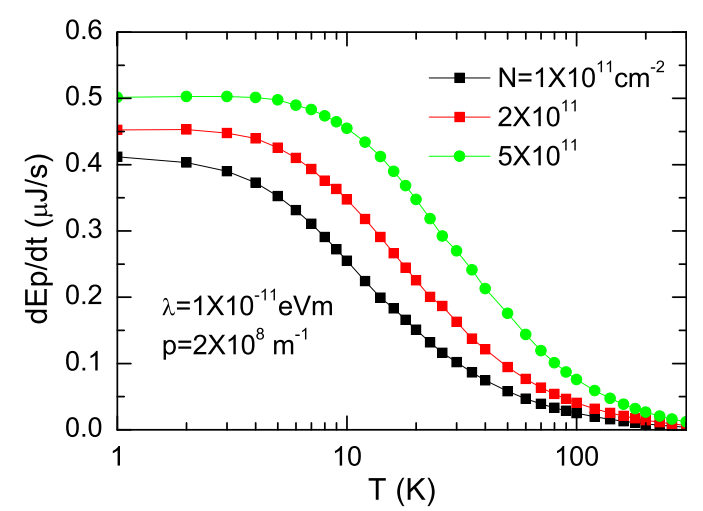

FIG. 2. Temperature dependence of ELR at various electron concentrations. 
chemical potential at finite temperature. The second effect is more significant. As temperature increase, the phase space decreases rapidly, leading to a large reduction of ELR at high temperatures. At low temperature $(T<10 \mathrm{~K})$, the thermal energy is much less than the incident energy. As a result, ELR is insensitive the temperature change. The scattering of the incident particle by the $2 \mathrm{DS} / \mathrm{RSO}$ is proportional to the electron concentration of the 2DS/RSO and the coupling strength $g_{q}$ which decreases with the concentration due to the screening effect. Therefore increasing the electron concentration of $2 \mathrm{DS} / \mathrm{RSO}$ leads to an enhanced ELR, but it increases with the electron concentration slower than the linear dependence.

The mean scattering time of the particle in $2 \mathrm{DS} / \mathrm{RSO}$ is defined as

$$
\tau=\varepsilon_{p}\left(\frac{d \varepsilon_{p}}{d t}\right)^{-1}
$$

In Fig. 3, we plot the wavenumber dependence of the mean scattering time under various RSO parameters. The scattering is generally weaker (long $\tau$ ) for slow and faster particles. For slow particles, the energy is low as well as the ELR, resulting in a long scattering time. For fast particles, higher energy and large ELR also lead to a weak scattering. There exists an optimal incoming energy at which the scattering of the incident particle by the $2 \mathrm{DS} / \mathrm{RSO}$ is the strongest. The results suggest that the scattering time is dominated by ELR at low energy and by the incoming energy at high energy. The interplay of the incoming energy and ELR results in a turn around scattering time at an intermediate energy. The optimal momentum where the scattering is strongest is dependent on the RSO parameter. A low $\lambda$ corresponds to lower ELR, thus a lower optimal momentum.

The diffusion constant is given by

$$
D_{\boldsymbol{p}} \sim(m p)^{2} \tau_{\boldsymbol{p}} \sim \varepsilon_{\boldsymbol{p}} \tau_{\boldsymbol{p}}
$$

From the mean scattering time, we can deduce that under strong Rashba coupling, diffusion constant increases with energy slowly at low energy and rapidly at high energy. Under a weak Rashba coupling, the diffusion constant decreases slowly at low energy and increases rapidly at high energy.

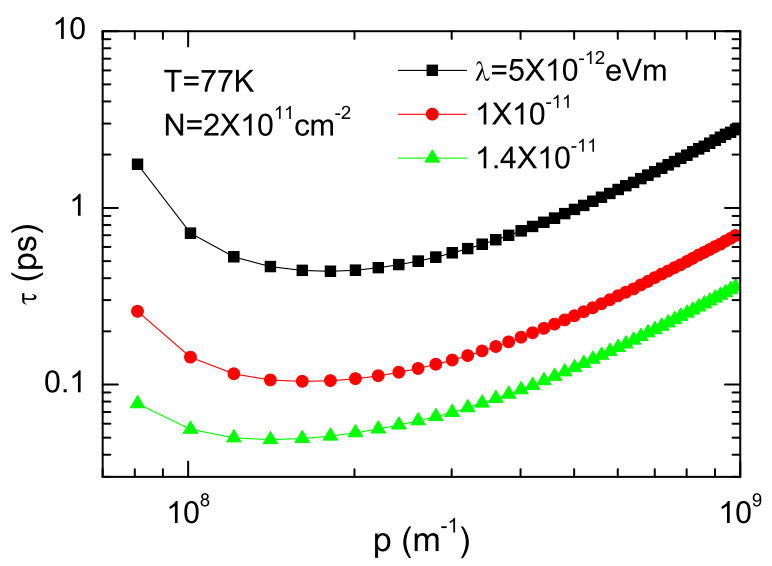

FIG. 3. Wavenumber dependence of the mean scattering time at various RSO parameters.
In conclusion, the energy-loss rate of a fast particle in 2DS/RSO has been calculated. Our method takes into account the full temperature and density dependence of the electronic polarizability, the scattering phase space, and the chemical potential. Therefore the result is valid at arbitrary temperatures. The momentum dependent scattering time shows the interplay of the incident energy and electronic transitions in 2DS/RSO. Experimentally, the RSO parameter can be tuned with an applied electrical field. This suggests that our result of $\lambda$-dependent ELR can be tuned under an applied field. The scattering time shown in Fig. 3 indicates that it can be tuned by one to two orders of magnitude when the RSO parameter is varied by a factor of around 3. This finding offers a method to design and modulate the transport of charged particles in semiconductors with finite Rashba spin-orbit coupling.

We thank Yee Sin Ang for stimulating discussion on ELR. W. Feng Acknowledges support by Jiangsu University Initial Funding for Advanced Talents (Grant No. 11JDG037). The work is partly supported by the Australia Research Council, by the 863 Program of China (No. 2011AA010205), the National Natural Science Foundation of China (Nos. 61131006 and 61021064), the Major National Development Project of Scientific Instrument and Equipment (No. 2011YQ150021), the Important National Science and Technology Specific Projects (No. 2011ZX02707), the major project (No. YYYJ-1123-1) of the Chinese Academy of Sciences, the Shanghai Municipal Commission of Science and Technology (No. 10JC1417000), and by CAS International Collaboration and Innovation Program on High Mobility Materials Engineering.

${ }^{1}$ S. A. Wolf, D. D. Awschalom, R. A. Buhrman, J. M. Daughton, S. von Molnár, M. L. Roukes, A. Y. Chtchelkanova, and D. M. Treger, Science 294, 1488 (2001).

${ }^{2}$ B. Datta and S. Das, Appl. Phys. Lett. 56, 665 (1990).

${ }^{3}$ X. F. Wang, P. Vasilopoulos, and F. M. Peeters, Phys. Rev. B 65, 165217 (2002).

${ }^{4}$ T. Koga, J. Nitta, H. Takayanagi, and S. Datta, Phys. Rev. Lett. 88, 126601 (2002).

${ }^{5}$ Th. Schäpers, G. Engels, J. Lange, Th. Klocke, M. Hollfelder, and H. Lüth, J. Appl. Phys. 83, 4324 (1998).

${ }^{6}$ E. I. Rashba, Fiz. Tverd. Tela (Leningrad) 2, 1224 (1960) [Sov. Phys. Solid State 2, 1109 (1960)]; E. I. Rashba and V. I. Sheka, in Landau Level Spectroscopy, edited by G. Landwehr and E. I. Rashba (North-Holland, Amsterdam, 1991), Vol. 1, p. 131.

${ }^{7}$ Y. Sato, T. Kita, S. Gozu, and S. Yamada, J. Appl. Phys. 89, 8017 (2001); D. Grundler, Phys. Rev. Lett. 84, 6074 (2000).

${ }^{8}$ J. Nitta, T. Akazaki, and H. Takayanagi, Phys. Rev. Lett. 78, 1335 (1997).

${ }^{9}$ J. Schliemann, J. C. Egues, and D. Loss, Phys. Rev. B 67, 085302 (2003).

${ }^{10}$ C. Zhang and Z. S. Ma, Phys. Rev. B 71, 121307 (2005).

${ }^{11}$ V. K. Dugaev, M. Inglot, E. Ya. Sherman, J. Berakdar, and J. Barnas, Phys. Rev. Lett. 109, 206601 (2012).

${ }^{12}$ S. Caprara, F. Peronaci, and M. Grilli, Phys. Rev. Lett. 109, 196401 (2012).

${ }^{13}$ D. Bercioux, D. F. Urban, F. Romeo, and R. Citro, Appl. Phys. Lett. 101, 122405 (2012).

${ }^{14}$ M. Esmaeilzadeh and S. Ahmadi, J. Appl. Phys. 112, 104319 (2012).

${ }^{15}$ M. Dey, S. K. Maiti, and S. N. Karmakar, J. Appl. Phys. 112, 024322 (2012).

${ }^{16}$ T. Eberlein, U. Bangert, R. R. Nair, R. Jones, M. Gass, A. L. Bleloch, K. S. Novoselov, A. Geim, and P. R. Briddon, Phys. Rev. B 77, 233406 (2008).

${ }^{17}$ J. Lu, K. P. Loh, H. Huang, W. Chen, and A. T. S. Wee, Phys. Rev. B 80, 113410 (2009).

${ }^{18}$ C. Zhang and Y. Takahashi, Phys. Rev. B 46, 9247 (1992).

${ }^{19}$ C. Zhang, N. Tzoar, and P. M. Platzman, Phys. Rev. B 37, 7326 (1988). 